\title{
Unexpected Peutz-Jeghers Syndrome in an Adult Presenting with Intermittent Upper Intestinal Obstruction. A Case Report
}

\author{
Paula Szanto ${ }^{1,2}$, Valentina Barbieru, ${ }^{1,2}$, Radu Badea ${ }^{2,3}$, Teodora Pop ${ }^{1,2}$, Ioana Rusu ${ }^{4}$, Nadim al Hajjar ${ }^{1,5}$
}

\author{
1) Department of \\ Gastroenterology, Regional \\ Institute of Gastroenterology \\ and Hepatology Prof. Dr. \\ Octavian Fodor \\ 2) $3^{\text {rd }}$ Medical Clinic, Iuliu \\ Hatieganu University of \\ Medicine and Pharmacy \\ 3) Department of \\ Ultrasonography, Regional \\ Institute of Gastroenterology \\ and Hepatology Prof. Dr. \\ Octavian Fodor \\ 4) Department of \\ Morphopathology, Regional \\ Institute of Gastroenterology \\ and Hepatology Prof. Dr. \\ Octavian Fodor \\ 5) $3^{\text {rd }}$ Surgical Clinic, Iuliu \\ Hatieganu University of \\ Medicine and Pharmacy \\ Cluj-Napoca, Romania
}

\author{
Address for correspondence: \\ Valentina Barbieru \\ $3^{\text {rd }}$ Medical Clinic \\ 19-21 Croitorilor Street \\ 400162 Cluj-Napoca \\ Romania
}

valentina.barbieru@yahoo.com

Received: 28.10.2013

Accepted: 17.11.2013

\begin{abstract}
Peutz-Jeghers syndrome is an autosomal dominant inherited disease, belonging to the hamartomatous polyposis syndromes. It is characterized by multiple hamartomatous polyps of the gastrointestinal tract associated with oral and anal mucocutaneous pigmentations. We report the case of an adult patient diagnosed with an atypical form of Peutz-Jeghers syndrome, thereby emphasizing the different possible syndrome phenotypes and the difficulty of their diagnosis.
\end{abstract}

Key words: small bowel obstruction - intussusception - jejunal polyps - Peutz-Jeghers syndrome.

\section{INTRODUCTION}

Peutz-Jeghers syndrome (PJS) is an autosomal dominant inherited disease, which belongs to the hamartomatous polyposis syndromes [1]. It is characterized by multiple hamartomatous polyps of the gastrointestinal tract associated with oral and anal mucocutaneous pigmentations [2]. The genetic lesion responsible for the phenotypic expression is in $66-94 \%$ of the cases considered to be a mutation on the short arm of chromosome 19 (19p13.3) of STK11/LKB1. Abrogation of this tumor suppressor gene results in both polyposis along with probably a separate process, leading to tumorigenesis, and thus an increased risk of developing malignancies [3, $4]$. Penetrance of the gene mutation is variable, resulting in a spectrum of phenotypic manifestations (inconsistent number, localization of polyps, different presentation of the macules) [5].
We report the case of an adult patient diagnosed with an atypical form of PJS, and we emphasize the different possible PJS phenotypes and the difficulty of their diagnosis.

\section{CASE REPORT}

A 41-year old woman without any past medical history, besides 2 deliveries and 3 abortions, was admitted to our hospital complaining of intermittent episodes of nausea, vomiting and pain in the right upper abdominal quadrant and epigastrium. The symptoms had no relation with food intake. Bowel transit was normal with stools every 1-2 days. The presenting symptoms started about a year prior to presentation and were partially ameliorated by antacids, antispasmodics and pain relievers. A weight loss of $8 \mathrm{~kg}$ in this year was noted but with preserved appetite and periods of regaining weight. The patient came from a rural area, worked in a botanical garden and denied any exposure to environmental or professional toxics.

The clinical exam revealed a slim woman with a BMI of $20.7 \mathrm{~kg} / \mathrm{m}^{2}$, pale, with dry mucosae, café-au-lait plaques on the skin of the upper trunk (similar to pityriasis versicolor). The abdomen was elastic, mobile with respiration, presenting tenderness in the epigastrium and the right upper quadrant.

Laboratory data showed a mild iron deficiency anemia (hemoglobin $11.2 \mathrm{~g} / \mathrm{dl}$ ), hypertriglyceridemia, hypocalcemia and a moderate inflammatory syndrome. No other blood, urine or stool changes were detected.

Esogastroduodenoscopy revealed a gastric atrophic mucosa with micropolyps of $0.2 \mathrm{~cm}$ (Fig.1), multiple erosions 
in the second part of the duodenum (DII) and an important duodeno-gastric reflux. Helicobacter pylori was negative. The same aspect had been found several months before in another service.

The abdominal ultrasound discovered a mass in the right upper quadrant that was suggestive for an entero-enteral intussusception with a circumferentially thickened wall $(10 \mathrm{~mm})$, raising the suspicion of a giant polypoid tumor in the small bowel (Fig.2). Bowel changes were also found in the right and left iliac fossa. The Doppler examination identified a possible tumoral mass of $20 / 20 \mathrm{~mm}$, vascularized through a branched pedicle, and considered to be part of the proximal jejunum. The bowels had normal peristalsis and parietal vascularization. No sign of ischemia was identified. These findings were confirmed by contrast enhanced ultrasound examination.

The abdominal computer tomography (CT) described the intestinal intussusception of the first jejunal loop (size $149 / 60 / 78 \mathrm{~mm}$ ) but did not bring any additional information to the underlying cause (Fig.3). Small mesenteric adenopathies were present.

A spiral enteroscopy was considered. Inspection of the entire jejunum identified two polyps. The first lesion was located at the junction of DIII with the first jejunal loop, it had about $40 \mathrm{~mm}$ and was the cause of the invagination (Fig. 4).

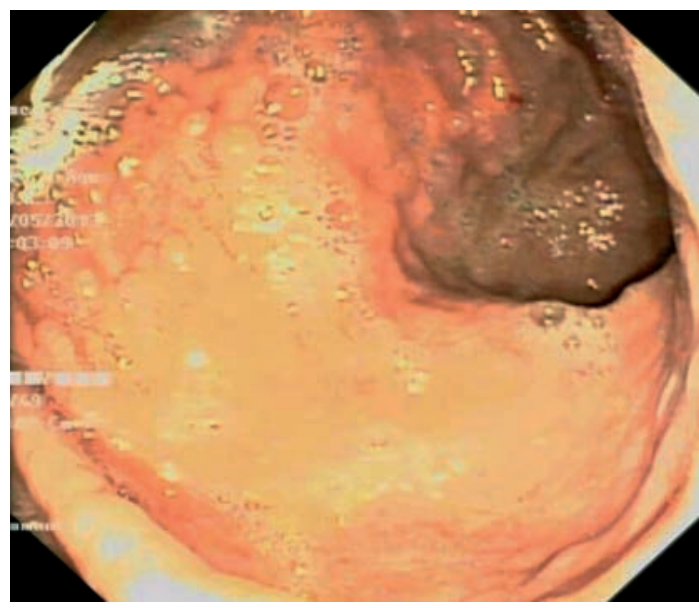

Fig. 1. Esophagogastroduodenoscopy: gastric micropolyps (histopathology: chronic nonspecific inflammation).

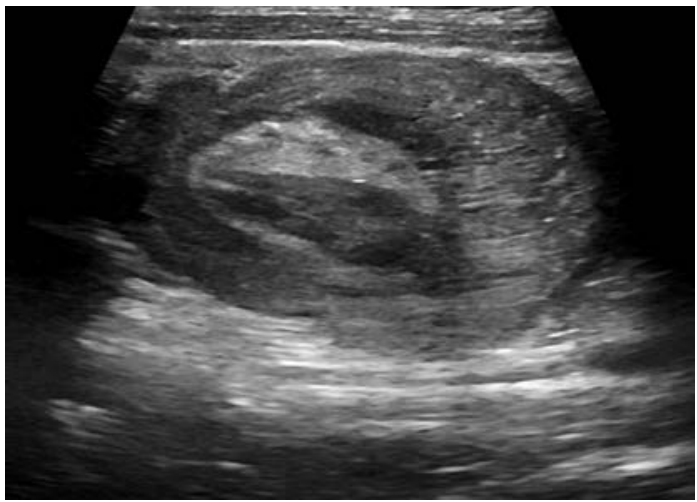

Fig. 2. Abdominal ultrasound: jejuno-jejunal intussusception.

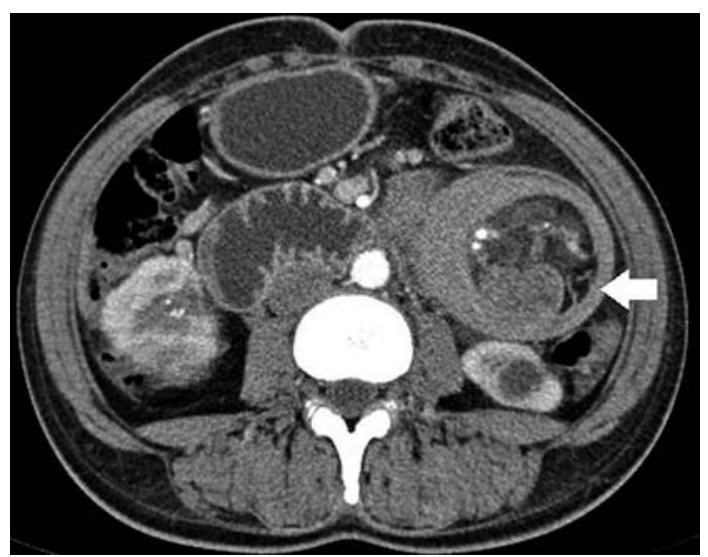

Fig. 3. Axial contrast-enhanced abdominal computer tomography (CT): jejuno-jejunal intussuscepion (arrow).

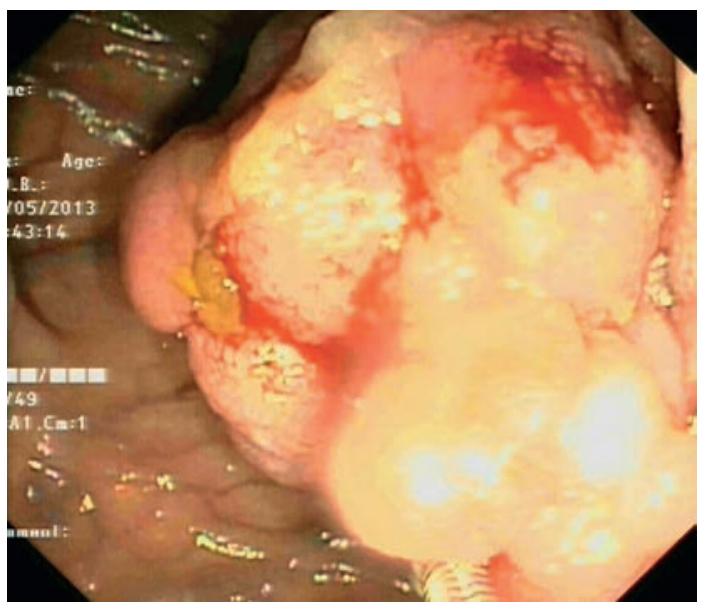

Fig. 4. Spiral-enteroscopy: a giant polyp $(4 \mathrm{~cm})$ in the proximal jejunum.

Another $40 \mathrm{~mm}$ polypoid tumor with ulcerations was found in the terminal jejunum. The procedure was both diagnostic (multiple biopsies taken from the polyps) and therapeutic, performing reduction of the intussusception. Additionally, colonoscopy was performed and no lesion was found.

Tumor markers were checked: CEA was normal, but CA125 was elevated $(101.6 \mathrm{IU} / \mathrm{ml})$.

Immediately after enteroscopy the patient had no complaints, but the day after the procedure, symptoms of small bowel obstruction (colicative pain in the right upper quadrant, nausea and vomiting) appeared. The patient was transferred to the surgical department. A double small bowel segmentary resection with termino-terminal anastomosis was performed (Fig. 5). Considering the findings, an intestinal polyposis syndrome was suspected and was sustained by the morphological description of the lesions as being hamartomatous polyps (Fig. 6). We add here that the first two histological exams, the gastric biopsy and the jejunum biopsy described nonspecific chronic inflammation.

In search of mucocutaneous lesions, an isolated oral pigmentation was found on the mucosa of the lower lip. No other lesions could be identified in the oral and anal region or on the hands and feet.

A further detailed family history was obtained. The patient had no recollection of her parents or brother having any gastro- 


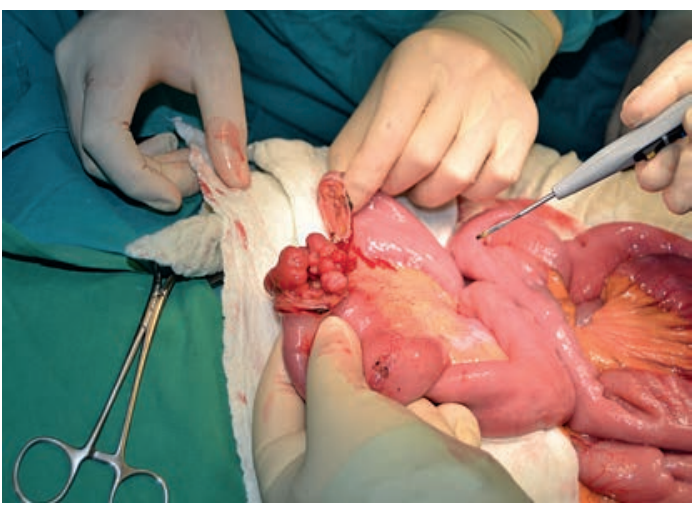

Fig. 5. Intraoperative aspect of the resected polyp.

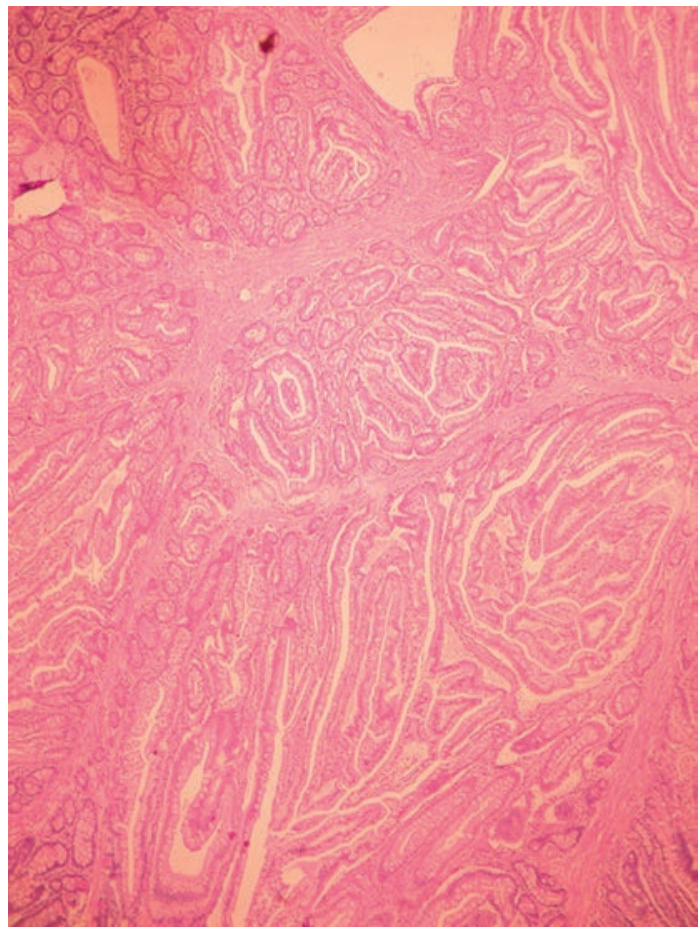

Fig. 6. Histopathological findings revealing hamartomatous characteristics of resected polyps (H\&E x40)

intestinal pathology, but her older sister had been investigated for colonic polyps.

The patient had an uneventful recovery and remained well 3 months later. Her asymptomatic children were also evaluated. They presented clinical features of PJS (dark macules around the oral area) and similar gastric micropolyposis. No polyps were found in the first part of the small bowel that was evaluated endoscopically. The patient and the children will be clinically and biologically reevaluated on a specific schedule. In the meantime a gynecological surveillance has been suggested and is mandatory, taking into consideration the elevated CA125 and the association between PJS and gynecological cancers.

\section{DISCUSSION}

Peutz-Jeghers syndrome, also known as hereditary intestinal polyposis syndrome is diagnosed according to the WHO criteria when the presence of mucocutaneous pigmentation, bowel hamartomatous polyps and a family history of PJS are found simultaneously [6]. Polyps found in PJS are commonly present in adolescence and early adulthood $[7,8]$. The median time to symptoms onset and first presentation with polyps is age 11-13 years and most individuals present symptoms by 20 years [9-11]. Anemia, rectal bleeding, abdominal pain, obstruction and intussusception are commonly presenting symptoms $[12,13]$. Because of the polyps, nearly $50 \%$ of the patients experience small intestine intussusception during lifetime [14]. Our patient was 41 year-old and her health problems had started only 1 year before presentation with symptoms suggestive of intermittent small bowel obstruction. Even if jejunum and ileum are known to be the most frequently involved parts of the gastrointestinal tract, colonoscopy is part of the diagnostic investigation. No colon polyps were found in our patient. In most cases, multiple segmental resections are mandatory. Combined endoscopic and surgical treatment has been reported and is considered to be the best in terms of quality of life $[15,16]$. Considering our patient, because of the size of the two jejunal polyps and especially because of the acute intestinal obstruction, surgery was the preferred therapy.

Melanotic macules on the lips and buccal mucosa are a cardinal feature, thus playing an important role in the early diagnosis. Usually the hyperpigmentations appear in the first year of life but in adulthood can fade and in some cases even disappear [6]. Therefore, the absence of melanin spots in an adult patient should not rule out the diagnosis of PJS. On the other hand, studies suggest that the mucosa of the lower lip is almost invariably involved [17], as in our patient, who had in this area her only mucosal spot. Considering the WHO criteria for PJS diagnosis: (I) three or more histologically confirmed PJ polyps (PJPs); (II) any number of PJPs with a family history of PJS; (III) characteristic, prominent, mucocutaneous pigmentations with a family history of PJS; (IV) any number of PJPs and characteristic, prominent, mucocutaneous pigmentation; none of the features were totally fulfilled in our case at the time of polyps diagnosis. The advanced age and the lack of typical phenotypic appearance did not suggest the diagnosis of PJS as the first option. Moreover, the first biopsies did not raise suspicion towards it. The diagnosis came forward only after enteroscopy and was strengthened by the histological examination and by the dominant transmission to the descendants.

Peutz-Jeghers syndrome is highly penetrant with variable expression. Only one case of non-penetrance of an LKB1 mutation has been reported so far [18]. De novo mutations are described as well [5]. Moreover, variability in the timing and number of mucocutaneuos pigmentations, polyps and cancer is highly unpredictable [6]. This is why, even if genetic testing is usually not necessary [6], analysis of germline STK11/LKB1 mutations may be necessary in any case of PJP which does not fulfill the WHO criteria.

\section{CONCLUSION}

Patients with PJS should be followed by a multidisciplinary team. Patients should be educated on the potential symptoms of intestinal obstruction and instructed on the need for cancer surveillance. Counseling and testing of asymptomatic family 
members is mandatory in order to prevent complications, including malignancy.

Conflicts of interest: None to declare.

\section{REFERENCES}

1. Arber N, Moshkowitz M. Small bowel polyposis syndromes. Curr Gastroenterol Rep 2011;13:435-441.

2. Giardiello FM, Brensinger JD, Tersmette AC, et al. Very high risk of cancer in familial Peutz-Jeghers syndrome. Gastroenterology 2000;119:1447-1453.

3. Hemminki A, Markie D, Tomlinson I, et al. A serine/threonine kinase gene defective in Peutz-Jeghers syndrome. Nature 1998;391:184-187.

4. Mehenni H, Blouin JL, Radhakrishna U, et al. Peutz-Jeghers syndrome: confirmation of linkage to chromosome 19p13.3 and identification of a potential second locus, on 19q13.4. Am J Hum Genet 1997;61:13271334.

5. Hernan I, Roig I, Martin B, Gamundi MJ, Martinez-Gimeno M, Carballo M. De novo germline mutation in the serine-threonine kinase STK11/LKB1 gene associated with Peutz-Jeghers syndrome. Clin Genet 2004;66:58-62.

6. Riegert-Johnson D, Gleeson FC, Westra W, et al. Peutz-Jeghers Syndrome. In: Riegert-Johnson DL, Boardman LA, Hefferon T, et al. (eds). Cancer Syndromes [Internet]. Bethesda (MD): National Center for Biotechnology Information (US); 2009. Available from: http://www. ncbi.nlm.nih.gov/books/NBK1826/.

7. Perzin KH, Fenoglio CM, Pascal RR. Tumors of the small and large intestine. In: Principles and Practice of Surgical Pathology. John Wiley and Sons, New York 1983:899-936.
8. Hearle N, Schumacher V, Menko FH, et al. Frequency and spectrum of cancers in the Peutz-Jeghers syndrome. Clin Cancer Res 2006;12:32093215.

9. Amos CI, Keitheri-Cheteri MB, Sabripour M, et al. Genotype-phenotype correlations in Peutz-Jeghers syndrome. J Med Genet 2004;41:327-333.

10. Gammon A, Jasperson K, Kohlmann W, Burt RW. Hamartomatous polyposis syndromes. Best Pract Res Clin Gastroenterol 2009;23:219231.

11. Schreibman IR, Baker M, Amos C, McGarrity TJ. The hamartomatous polyposis syndromes: a clinical and molecular review. Am J Gastroenterol 2005;100:476-490.

12. Zbuk KM, Eng C. Hamartomatous polyposis syndromes. Nat Clin Pract Gastroenterol Hepatol 2007;4:492-502.

13. Gammon A, Jasperson K, Kohlmann W, Burt RW. Hamartomatous polyposis syndromes. Best Pract Res Clin Gastroenterol 2009;23:219231.

14. Hinds R, Philp C, Hyer W, Fell JM. Complications of childhood Peutz-Jeghers syndrome: implications for pediatric screening. J Pediatr Gastroenterol Nutr 2004; 39:219-220.

15. Lin BC, Lien JM, Chen RJ, Fang JF, Wong YC. Combined endoscopic and surgical treatment for the polyposis of Peutz-Jeghers syndrome. Surg Endosc 2000;14:1185-1187.

16. Seenath MM, Scott MJ, Morris AI, Ellis A, Hershman MJ. Combined surgical and endoscopic clearance of small-bowel polyps in PeutzJeghers syndrome. J R Soc Med 2003;96:505-506.

17. Westerman AM, Entius MM, de Baar E, et al. Peutz-Jeghers syndrome: 78-year follow-up of the original family. Lancet 1999;353:1211-1215.

18. Olschwang S, Markie D, Seal S, et al. Peutz-Jeghers disease: most, but not all, families are compatible with linkage to 19p13.3. J Med Genet 1998;35:42-44. 